\title{
Impact of Grain Boundary Density on Oxide Scaling Revisited
}

\author{
Christine Geers $^{1} \cdot$ Itai Panas ${ }^{1}$
}

Received: 6 February 2018 / Published online: 28 August 2018

(c) The Author(s) 2018

\begin{abstract}
A straightforward conceptual tool for discriminating between different oxide scaling processes deviating from the parabolic standard model is formulated. Grain boundary diffusion-controlled oxide scaling is generalized to include lateral grains coarsening. Building on traditional Wagner theory, attenuation of rates of inwards growing oxides owing to the gradual loss of grain boundary density is revisited. Two viable cases are identified. One has the rate of grain boundary density loss to be independent of the rate of oxide growth, while the second case takes the two instantaneous rates to be equal. Simple parabolic-logarithmic and superparabolic-cubic expressions are arrived at for the two cases, respectively. Usefulness is demonstrated by applying the models to published experimental data from 1990 to date. Upon arrival at the superparabolic-cubic behaviour, a generic mathematical form analogous to a 'spring force' attenuating the scale growth was identified. 'Parabolic', 'cubic' and 'logarithmic' scaling emerges as limiting cases.
\end{abstract}

Keywords Oxidation kinetics $\cdot$ Model $\cdot$ Scaling $\cdot$ Grain boundary transport

\section{Introduction}

For several decades, the outstanding properties of oxide dispersion strengthened alloys have served as a vehicle for fundamental research at the forefront of industrial materials development of relevance to chemical engineering and energy conversion processes $[1,2]$. The efficient generic high-temperature oxide scaling of aluminaforming ODS alloys-i.e. slow growing and well adhering-apparently contradicts their complex and diverse compositions and textures. However, it should be kept in mind that the complexities of said class of alloys - they comprise a base metal, e.g. $\mathrm{Co}, \mathrm{Fe}(\mathrm{Ni})$ or $\mathrm{Ni}(\mathrm{Co})$, a scale-forming component $(\mathrm{Al})$ and commonly chromium

Christine Geers

geersc@chalmers.se

1 Department of Chemistry and Chemical Engineering, Chalmers University of Technology, Kemivägen 10, 41296 Gothenburg, Sweden 
providing the third element effect [3-6] — are actually tuned to achieve the sought near-ideal long-term scaling property. Additional fine-tuning, mainly aiming at improved scale/alloy adhesion, is achieved by the so-called reactive elements-e.g. Y, La, Zr, Hf - often appearing as submicron oxide particles in the ODS alloy [7, 8]. Beneficial impact has been correlated with change in electronic structure of oxide grain boundaries [9], and avoidance of scale spallation due to sulphur decoration at alloy-oxide interface by REs acting sulphur getters in the alloy [10]. Currently, the origins of often near-ideal oxidation properties at high temperatures are increasingly being unravelled and revisited by employing state-of-the-art analysis on the nanoscale in conjunction with atomistic first-principles modelling [11].

Inevitably, such emerging novel perspectives attract renewed interest in generic models for the transformation from early transient oxide formation to late ideal oxide growth kinetics in order to offer a semi-quantitative conceptual framework for alloys development in general. Building on Fickian diffusion, today still, the contemporary overall understanding of the oxidation of metals is based on the groundbreaking works of Tammann [12] and Wagner [13]. Also the decisive impact of grain boundaries, constituting easy paths for ion diffusion has long been recognized [14-16]. A recent attempt at connecting oxide grains coarsening to the oxide scaling as measured by inspecting oxide scale cross sections $[17,18]$ has served inspiration to the present effort. Thus, the objective of this study is to expose generic origins of near-ideal oxidation properties, so as to distinguish between beneficial oxide scaling and corrosion. To this end, two models are derived representing two different physical realities in that a particular alloy is taken to obey either 'superparabolic-cubic' or 'parabolic-logarithmic' short-term-long-term oxide scaling kinetics. Experimental data, from 1990 till present, form basis for the following discussion. As a consequence, no statistical analyses are provided here. It is, however, greatly encouraged, in conjunction with modelling, in view of the current limited predictive capacity of alloy development beyond empirical knowledge of the oxidation behaviour [19].

\section{Modelling Oxide Grain Coarsening During Scale Growth}

The present effort relies on a clear distinction between corrosion and oxide scale formation. First and foremost, the formation of an adherent oxide scale acts to prevent breakaway corrosion. Also, sacrificing metal to form a barrier oxide often offers the alloy protection against a hostile environment. And while corrosion is often specific to the particular application, we understand the build-up of a protective oxide scale to be more generic in nature. In this spirit, below we explore to what extent grain boundary transport alone - as attenuated by change of grain boundary density exclusively taking place at the mobile reaction zone-may suffice to describe experimentally observed oxide scale growth kinetics. Thus, it is implied below that first-order kinetics in loss of grain boundary density alone is indeed insufficient in reproducing experiment, owing to unphysical limiting properties. This is in contrast to the two expressions at focus in the present study, emerging from second-order kinetics. 
In what follows, to accentuate novel features, our approach draws inspiration from Smeltzer et al. [15], Naumenko et al. [17] and Young et al. [18]. Thus, the effective diffusivity of oxygen ions is subdivided into lattice and grain boundary components

$$
D_{\text {eff }}=D_{\mathrm{L}}(1-f)+D_{\mathrm{GB}} f=D_{\mathrm{L}}\left(1+\frac{D_{\mathrm{GB}}-D_{\mathrm{L}}}{D_{\mathrm{L}}} f\right) \text { where } f(t)=\frac{2 \delta_{\mathrm{GB}}}{r_{\mathrm{G}}},
$$

$\delta_{\mathrm{GB}}$ is the width of a grain boundary and $r_{E}(t)$ is the lateral length of a growing grain. For the diffusion flux, it follows that

$$
J_{\mathrm{O}}=\frac{1}{R T} \frac{\mathrm{d} \mu_{O}}{\mathrm{~d} x} C_{\mathrm{O}} D_{\mathrm{L}}\left(1+\frac{D_{\mathrm{GB}}-D_{\mathrm{L}}}{D_{\mathrm{L}}} f\right)
$$

The first of the two fundamental assumptions of the present study is that the oxide scale microstructure evolution takes place exclusively at the moving oxide front. It is discretized according to the so-called low-gradient approximation

$$
\bar{J}_{\mathrm{O}}=\frac{1}{R T} \frac{\Delta \mu_{\mathrm{O}}}{X} C_{\mathrm{O}} D_{\mathrm{L}}\left(1+\frac{D_{\mathrm{GB}}-D_{\mathrm{L}}}{D_{\mathrm{L}}} f\right)
$$

where $X$ is the instantaneous scale thickness. Equation 2 b may subsequently be converted into a scale growth velocity by writing

$$
\frac{\mathrm{d} X}{\mathrm{~d} t}=\bar{J}_{\mathrm{O}} V_{\mathrm{O}}
$$

where $V_{\mathrm{O}}$ is the specific volume of the mobile species, such that $C_{\mathrm{O}} V_{\mathrm{O}}=1$. The differential equation to solve becomes (see, for example, [11])

$$
\frac{\mathrm{d} X}{\mathrm{~d} t}=\frac{1}{R T} \frac{\Delta \mu_{\mathrm{O}}}{X} D_{\mathrm{L}}\left(1+\frac{D_{\mathrm{GB}}-D_{\mathrm{L}}}{D_{\mathrm{L}}} f\right) .
$$

Let $C_{\mathrm{GB}}=\frac{1}{r_{\mathrm{G}}}$ be the lateral grain boundary density; then by virtue of $f=\frac{2 \delta_{\mathrm{GB}}}{r_{\mathrm{G}}}(c f$. Equation 1)

$$
\frac{\mathrm{d} X}{\mathrm{~d} t}=\frac{\Delta \mu}{R T} \frac{1}{X} D_{\mathrm{L}}\left(1+\frac{D_{\mathrm{GB}}-D_{\mathrm{L}}}{D_{\mathrm{L}}} 2 \delta_{\mathrm{GB}} C_{\mathrm{GB}}\right)
$$

One approach is to take the impact of loss of grain boundary density $C_{\mathrm{GB}}$ on scale growth to exhibit first-order kinetics, $C_{\mathrm{GB}}(t)=C_{\mathrm{GB}}(0) \mathrm{e}^{-k t}$, and hence, $f(t)=f_{0} \mathrm{e}^{-k t}=\frac{2 \delta_{\mathrm{GB}}}{r_{\mathrm{o}}} \mathrm{e}^{-k t}$. In this case, Eq. 4 becomes

$$
\frac{\mathrm{d} X}{\mathrm{~d} t}=\frac{1}{R T} \frac{\Delta \mu_{\mathrm{O}}}{X} D_{\mathrm{L}}\left(1+\frac{D_{\mathrm{GB}}-D_{\mathrm{L}}}{D_{\mathrm{L}}} f_{0} \mathrm{e}^{-k t}\right)
$$


from which it follows that

$$
X=\left\{2 \frac{\Delta \mu_{\mathrm{O}}}{R T} D_{\mathrm{L}}\left(t+\frac{D_{\mathrm{GB}}-D_{\mathrm{L}}}{D_{\mathrm{L}} k} f_{0}\left[1-\mathrm{e}^{-k t}\right]\right)\right\}^{\frac{1}{2}}
$$

Now, let the grain boundary diffusion be the dominating transport channel for oxygen ions, i.e. $D_{\mathrm{GB}} f \gg D_{\mathrm{L}}(1-f)$. Indeed, the second fundamental assumption of the present study is that oxide scaling owing to lattice diffusion is vanishingly small. This is in order to unravel the impact of grains coarsening on subparabolic limiting scale growth, ubiquitous to oxidation of metals. Inasmuch as Eq. 5b displays parabolic limiting scale growth owing to the lattice diffusion channel, in its absence, the first-order kinetics model

$$
X=\left\{2 \frac{\Delta \mu_{\mathrm{O}}}{R T}\left(\frac{D_{\mathrm{GB}}}{k} f_{0}\left[1-\mathrm{e}^{-k t}\right]\right)\right\}^{\frac{1}{2}}
$$

must be discarded because it predicts an unphysical constant limiting scale thickness for $t \rightarrow \infty$. Here, we proceed by considering second-order kinetics $\frac{\mathrm{d} C_{\mathrm{GB}}}{\mathrm{d} t}=-k C_{\mathrm{GB}}^{2}$ for the decay of grain boundary density, as was originally proposed by Fehlner and Mott [16]. They obtained $C_{\mathrm{GB}}=\frac{C_{\mathrm{GB}}^{0}}{1+C_{\mathrm{GB}}^{0} k \cdot t}=\frac{1}{r_{0}+k \cdot t}$, i.e. $f=\frac{2 \delta_{\mathrm{GB}}}{r_{0}+k \cdot t}$ for $r_{\mathrm{G}}(t)=r_{0}+k \cdot t$. Furthermore, as a consequence of $D_{\mathrm{L}} \rightarrow 0$, Eq. 4 can be modified to read

$$
\frac{\mathrm{d} X}{\mathrm{~d} t}=\frac{\Delta \mu}{R T} \cdot \frac{1}{X} \cdot \frac{2 \delta_{\mathrm{GB}} D_{\mathrm{GB}}}{r_{0}+k \cdot t}
$$

At this point, our deduction bifurcates as we may have the grains coarsening to be either implicitly or explicitly connected to the rate of scale growth (see the "Parabolic-Logarithmic Oxide Growth Mode" and "Superparabolic-Cubic Oxide Growth Mode" sections, respectively).

\section{Parabolic-Logarithmic Oxide Growth Mode}

Let the rate of grains coarsening $k$ be nonzero constant and independent of the growth rate of the oxide scale, noting that $k=0$ constitutes the parabolic oxide growth limit. Hence, for $k \neq 0$ we write

$$
r_{\mathrm{G}}=r_{0}+k t=r_{0}\left(1+\frac{k}{r_{0}} t\right)=r_{0}\left(1+\frac{t}{t_{0}}\right)
$$

to obtain the corresponding time dependence of scale thickness

$$
X=\left\{2 \frac{D_{\mathrm{GB}} 2 \delta_{\mathrm{GB}}}{k} \frac{\Delta \mu}{R T} \ln \left[1+\frac{k}{r_{0}} t\right]\right\}^{\frac{1}{2}}=\left\{2 \frac{D_{\mathrm{GB}} 2 \delta_{\mathrm{GB}}}{k} \frac{\Delta \mu}{R T} \ln \left[1+\frac{t}{t_{0}}\right]\right\}^{\frac{1}{2}}
$$


It follows from Eqs. 7 and 8a that

$$
r_{\mathrm{G}}=r_{0}\left(1+\frac{t}{t_{0}}\right)=r_{0} \exp \left[\frac{k X^{2}}{2 D_{\mathrm{GB}} 2 \delta_{\mathrm{GB}} \frac{\Delta \mu}{R T}}\right]
$$

as depicted in Fig. 1.

By virtue of Eq. 1, we identify $f=\frac{2 \delta_{\mathrm{GB}}}{r_{\mathrm{G}}}$ to obtain

$$
f=\frac{2 \delta_{\mathrm{GB}}}{r_{0}} \exp \left[-\frac{k X^{2}}{2 D_{\mathrm{GB}} 2 \delta_{\mathrm{GB}} \frac{\Delta \mu}{R T}}\right]
$$

\section{Parabolic-logarithmic model}

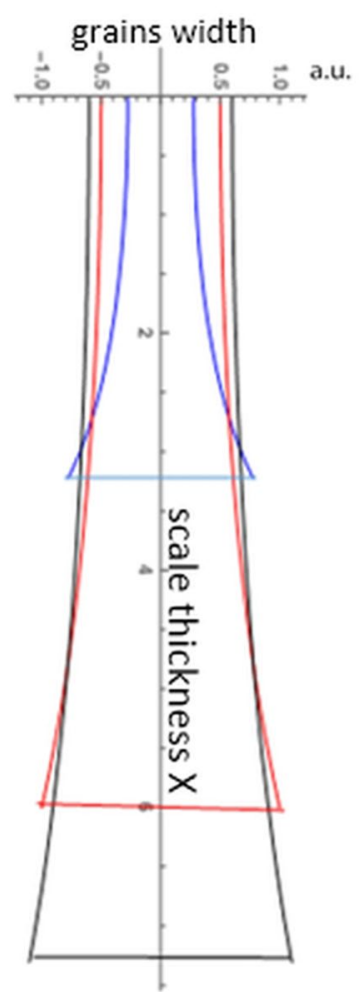

lowest $D_{G B}$, lowest $r_{0}$ intermediate $D_{G B}$, intermediate $r_{0}$

- highest $D_{G B}$, highest $r_{0}$

Fig. 1 Evolution of grain width as function of scale thickness for three different diffusivities and three different initial grain sizes (compare Eq. 8b). The curvatures reflect the constant velocities of grains coarsening eventually overtaking those of scale growth 
Thus, Eqs. 8b and 8c capture the impact of an accelerated effective grains coarsening process, corresponding to the resulting parabolic-logarithmic scaling in Eq. 8a. At this point, the generic form of the decelerating impact of $f$ in Eq. 1 is highlighted. Indeed, it can be understood to attenuate the parabolic scale growth by adding a second term to the activation energy for diffusion, this being quadratic in the oxide scale thickness $X$

$$
D_{\mathrm{GB}} f=D_{\mathrm{GB}}^{0} \frac{2 \delta_{\mathrm{GB}}}{r_{0}} \exp \left[-\left(\frac{E_{\mathrm{A}}}{R T}+\frac{k X^{2}}{2 D_{\mathrm{GB}} 2 \delta_{\mathrm{GB}} \frac{\Delta \mu}{R T}}\right)\right]
$$

By taking this term to represent the potential energy of a classical spring, the parabolic-logarithmic scaling Eq. 8a may intuitively be understood to result from a 'spring force', which resists parabolic scale growth. Indeed, rapidly decelerating scale growth may imply a significant long-term remnant (electro-)chemical potential gradient across the scale, which may in turn leave the scale vulnerable to scavenging local (electro-)chemical diffusion processes (see, for example, Geers et al. [20]).

Moreover, we note from Eq. $8 \mathrm{~b}$ that early into the oxidation process

$$
\frac{t}{t_{0}}=\frac{k}{2 D_{\mathrm{GB}} 2 \delta_{\mathrm{GB}} \frac{\Delta \mu}{R T}} X^{2}+\frac{1}{2}\left(\frac{k}{2 D_{\mathrm{GB}} 2 \delta_{\mathrm{GB}} \frac{\Delta \mu}{R T}}\right)^{2} X^{4}+\cdots
$$

Thus, we make contact with the parabolic limit, in that very early the first term in Eq. 9a is understood to dominate

$$
X \approx\left(2 \frac{D_{\mathrm{GB}} 2 \delta_{\mathrm{GB}}}{r_{0}} \frac{\Delta \mu}{R T}\right)^{\frac{1}{2}} \cdot \sqrt{t}
$$

The latter tells of oxide scale growth by grain boundary diffusion in the absence of grains coarsening.

\section{Superparabolic-Cubic Oxide Growth Mode}

Instead of a constant grains coarsening rate, here we assume that the instantaneous lateral grains coarsening velocity is proportional to the instantaneous rate of vertical oxide growth, suggestive of a grain boundary-mediated oxidation process which consumes grain boundary density, i.e.

$$
r_{\mathrm{G}}=r_{0}+k(X) \cdot t=k(X)\left(t_{0}^{c}+t\right) \equiv \alpha \frac{\mathrm{d} X}{\mathrm{~d} t}\left(t_{0}^{c}+t\right)
$$

In what follows, we give special meaning to $t_{0}$, i.e. the initial curing time $t_{0}^{c}$ for the grains coarsening that attenuates the scale growth. Also, we take $\alpha=1$ to say that its 
influence is absorbed by the material specific $D_{\mathrm{GB}} 2 \delta_{\mathrm{GB}}$. By inserting Eq. 10 into Eq. 3, we arrive at the corresponding time evolution of scale thickness

$$
X=\left(9 D_{\mathrm{GB}} 2 \delta_{\mathrm{GB}} t_{0}^{c} \frac{\Delta \mu}{R T}\right)^{1 / 3}\left[\sqrt{1+\frac{t}{t_{0}^{c}}}-1\right]^{2 / 3}
$$

Linking the grains coarsening rate to that of the scale growth puts Eq. (11) on par with the parabolic $t^{1 / 2}$ growth mode, in that besides the curing time $t_{0}^{c}$ no additional parameters are introduced.

Characteristics of the superparabolic-cubic oxide scaling may be deduced from the early and late time limits of Eq. 11. Thus, for $t \ll t_{0}^{c}$ we have the superparabolic $X \propto t^{\frac{2}{3}}$, i.e.

$$
X\left(t \ll t_{0}^{c}\right) \rightarrow\left(9 D_{\mathrm{GB}} 2 \delta_{\mathrm{GB}} t_{0}^{c} \frac{\Delta \mu}{R T}\right)^{1 / 3}\left(\frac{1}{2} \frac{t}{t_{0}^{c}}\right)^{2 / 3}
$$

while in the limit $t_{0}^{c} \ll t$ we obtain the cubic rate law,

$$
X\left(t \gg t_{0}^{c}\right) \rightarrow\left(9 D_{\mathrm{GB}} 2 \delta_{\mathrm{GB}} \frac{\Delta \mu}{R T}\right)^{1 / 3} t^{1 / 3}
$$

i.e. $X \propto t^{\frac{1}{3}}$ and independent of $t_{0}^{c}$.

Now we explore the time evolution of the effective grain size $r_{\mathrm{G}}(t)$. Thus, by differentiating Eq. 11 with respect to time, and for $t>0$ we have

$$
\frac{\mathrm{d} X}{\mathrm{~d} t}=\frac{1}{3}\left(9 D_{\mathrm{GB}} 2 \delta_{\mathrm{GB}} t_{0}^{c} \frac{\Delta \mu}{R T}\right)^{1 / 3} \frac{1 / t_{0}^{c}}{\sqrt{1+\frac{t}{t_{0}^{c}}}\left[\sqrt{1+\frac{t}{t_{0}^{c}}}-1\right]^{1 / 3}}
$$

Inserting Eq. 14 into Eq. 10, we obtain

$$
r_{\mathrm{G}}=\frac{1}{3}\left(9 D_{\mathrm{GB}} 2 \delta_{\mathrm{GB}} t_{0}^{c} \frac{\Delta \mu}{R T}\right)^{1 / 3} \frac{\sqrt{1+\frac{t}{t_{0}^{c}}}}{\left[\sqrt{1+\frac{t}{t_{0}^{c}}}-1\right]^{1 / 3}}
$$

We observe that for $t_{0}^{c} \gg t$ we have $r_{\mathrm{G}} \propto t^{-\frac{1}{3}}$

$$
r_{\mathrm{G}}\left(t_{0}^{c} \gg t\right)=\frac{1}{3}\left(9 D_{\mathrm{GB}} 2 \delta_{\mathrm{GB}} t_{0}^{c} \frac{\Delta \mu}{R T}\right)^{1 / 3} \frac{1}{\left[\frac{t}{2 t_{0}^{c}}\right]^{1 / 3}},
$$


and for $t_{0}^{c} \ll t$, we obtain $r_{\mathrm{G}} \propto t^{\frac{1}{3}}$ independent of $t_{0}^{c}$

$$
r_{\mathrm{G}}\left(t_{0}^{c} \ll t\right) \rightarrow \frac{1}{3}\left(9 D_{\mathrm{GB}} 2 \delta_{\mathrm{GB}} \frac{\Delta \mu}{R T}\right)^{1 / 3} t^{1 / 3}
$$

It is noted from Eq. 16 that for $t<t_{0}^{c}$ the grain boundary "curing" is controlled by the rapid initial oxide growth rate. Thus, from Eq. 16 it is inferred that $\frac{\mathrm{d} X}{\mathrm{~d} t} t_{0}^{c}\left(\equiv r_{0}\right.$, see Eq. 10) $\rightarrow \infty$ for $t \rightarrow 0$. Here, contact can be made with lattice diffusion driven single-crystal growth (see Eq. 1) in that this suggests the existence of an initial grain boundary free oxide film, which initially at $t<t_{0}^{c}$ becomes increasingly fine grained as the oxide scale starts growing [21]. Thus, by allowing only grain boundary transport, the grain boundary density is forced to initially increase owing to the retardation term in

$$
\frac{\mathrm{d} r_{\mathrm{G}}}{\mathrm{d} t}=\frac{\mathrm{d}^{2} X}{\mathrm{~d} t^{2}}\left(t_{0}^{c}+t\right)+\frac{\mathrm{d} X}{\mathrm{~d} t}
$$

until reaching a maximum for a critical grain size $r_{\mathrm{G}}^{c}$ at $t=\frac{5 t_{0}^{c}}{4}$

$$
r_{\mathrm{G}}^{c}=r_{\mathrm{G}}\left(1.25 t_{0}^{c}\right) \approx 1.8899\left(\frac{1}{3} D_{\mathrm{GB}} 2 \delta_{\mathrm{GB}} t_{0}^{c} \frac{\Delta \mu}{R T}\right)^{\frac{1}{3}}
$$

Subsequently, the grain boundary density decays owing to the grains coarsening (Eq. 17), which in turn is the reason for the limiting cubic scale growth (cf. Equation 13, see Fig. 2a). Combining Eqs. 11 and 15, we arrive at the universal result

$$
r_{\mathrm{G}}=\frac{X}{3} \frac{\sqrt{1+\frac{t}{t_{0}^{c}}}}{\sqrt{1+\frac{t}{t_{0}^{c}}}-1}
$$

Naively, the ratio $\frac{r_{\mathrm{G}}}{X}$ can be used to explain the change in top angle $\theta$ of the pyramidal shaped grains which constitute the growing oxide scale [14] (see Fig. 2b). We obtain

$$
\theta=2 \tan ^{-1}\left[\frac{1}{6} \frac{\sqrt{1+\frac{t}{t_{0}^{c}}}}{\sqrt{1+\frac{t}{t_{0}^{c}}}-1}\right]
$$

In particular, it is noted that the top angle starts at $180^{\circ}$ at $t=0$ and also that the limiting ratio $\frac{r_{G}}{X}(t \rightarrow \infty)=\frac{1}{3}$ reflects a limiting top angle of $\sim 18.9^{\circ}$. At $=\frac{5 t_{0}^{c}}{4}$, i.e. where $r_{\mathrm{G}}=r_{\mathrm{G}}^{c}$, the ratio $r_{\mathrm{G}} / X$ reaches unity, corresponding to top angle of $\sim 53.13^{\circ}$. 


\section{Superparabolic-cubic model}
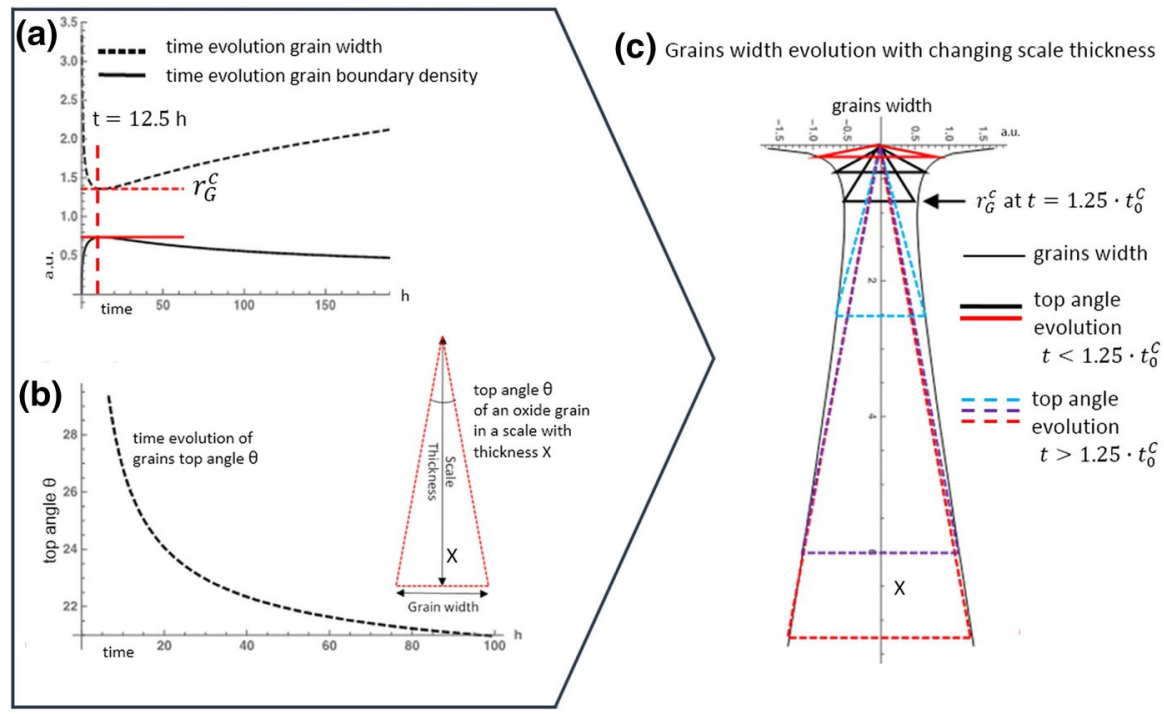

Fig. 2 Superparabolic-cubic model: Generic time evolution of grain width (Eq. 15) and grain boundary density with corresponding critical values for $t=1.25 \cdot t_{0}^{c}$ indicated, i.e. where the two functions reach their minima and maxima, respectively. Here, $10 \mathrm{~h}$ initial curing time is employed. $\mathbf{b}$ The universal oxide grains top angle evolution as function of oxide scaling time is displayed, Eq. 19b. $\mathbf{c}$ Evolution of grain width (horizontal, Eq. 15) with scale growth (vertical) (cf. Equation 11)

Finally, from Eq. 11 we have

$$
\left(9 D_{\mathrm{GB}} 2 \delta_{\mathrm{GB}} t_{0}^{c} \frac{\Delta \mu}{R T}\right)^{-1 / 2} X^{3 / 2}=\sqrt{1+\frac{t}{t_{0}^{c}}}-1
$$

This may be inserted into Eq. 19a to obtain

$$
r_{\mathrm{G}}=\frac{X}{3}\left[1+\left(9 D_{\mathrm{GB}} 2 \delta_{\mathrm{GB}} t_{0}^{c} \frac{\Delta \mu}{R T}\right)^{\frac{1}{2}} X^{-\frac{3}{2}}\right]
$$

which may be employed to identify $f=\frac{2 \delta_{\mathrm{GB}}}{r_{\mathrm{G}}}$ in Eq. 1. Instead, we proceed by inserting Eq. 19d into Eq. 6 to obtain

$$
\frac{\mathrm{d} X}{\mathrm{~d} t}=\frac{\Delta \mu}{R T} \cdot \frac{6 \delta_{\mathrm{GB}} D_{\mathrm{GB}}}{\left[X^{2}+\left(9 D_{\mathrm{GB}} 2 \delta_{\mathrm{GB}} t_{0}^{c} \frac{\Delta \mu}{R T}\right)^{\frac{1}{2}} X^{\frac{1}{2}}\right]}
$$


Subsequently, integrating this expression gives

$$
18 \delta_{\mathrm{GB}} D_{\mathrm{GB}} \frac{\Delta \mu}{R T} \cdot t=2\left(9 D_{\mathrm{GB}} 2 \delta_{\mathrm{GB}} t_{0}^{c} \frac{\Delta \mu}{R T}\right)^{\frac{1}{2}} X^{\frac{3}{2}}+X^{3}
$$

It is gratifying to note (1) how the two central asymptotes of the superparabolic-cubic model, Eqs. (12) and (13), come out clearly in Eq. 19f, as well as (2) the crucial role of $t_{0}^{c}$ as it controls the transition between said two asymptotes.

\section{Results and Discussion}

By comparing to the experimental literature, here we demonstrate the possible validity of the two fundamental assumptions - the predominance of grain boundary transport and the location of grains coarsening processes to the mobile reaction zone, vide supra-as well as the potential usefulness of the emerging conceptual understanding.

Throughout, connection between scale thickness $X$ and specific mass gain $\Delta m$ is made according to

$$
\Delta m=X \cdot \rho(\text { oxide })
$$

To this end, in the "Application of Superparabolic-Cubic Model" section we demonstrate the applicability of the superparabolic-cubic model

$$
X_{\mathrm{S}-\mathrm{C}}=\text { prefactor } \times\left[\sqrt{t+t_{0}^{c}}-\sqrt{t_{0}^{c}}\right]^{2 / 3}
$$

The "Application of Parabolic-Logarithmic Model" section focuses on the usefulness of the parabolic-logarithmic model

$$
X_{\mathrm{P}-\mathrm{L}}=\left\{\text { Constant } \times \ln \left[1+\frac{k t}{r_{0}}\right]\right\}^{\frac{1}{2}},
$$

while the concept of effective grains coarsening is introduced in the "Effective' Grain Boundary Density in Oxide Scaling" section in order to demonstrate the versatility of the approach.

\section{Application of Superparabolic-Cubic Model}

We start out by exploring the impact of the initial curing time $t_{0}^{c}$ on the time evolution of oxide scaling. Excellent agreement with the mass gain curve by Quadakkers et al. [22] is obtained for the alloy PM2000 at $1200{ }^{\circ} \mathrm{C}$ in air (Fig. 3), by setting the 


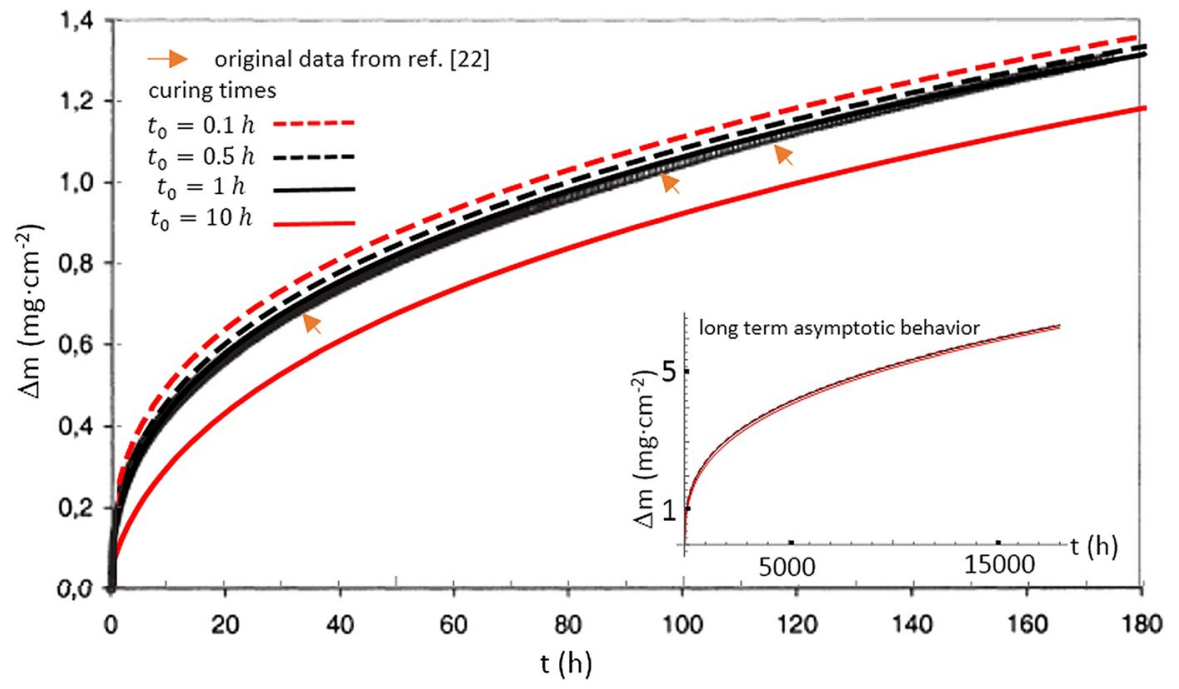

Fig. 3 Parameter sensitivity of superparabolic-cubic model is illustrated for initial curing times $t_{0}^{c}=0.1 \mathrm{~h}$ (red dashed); $0.5 \mathrm{~h}$ (black dashed); $1 \mathrm{~h}$ (full black); $10 \mathrm{~h}$ (full red) to compare with the experimental mass gain curve at $1200{ }^{\circ} \mathrm{C}$, from [22]. Best agreement is obtained for $t_{0}^{c}=1 \mathrm{~h}$. Inset: illustration of common asymptote irrespective of choice of $t_{0}^{c}$ (cf. Equation 13) (Color figure online)

prefactor to be $0.245 \mathrm{mg} / \mathrm{cm}^{2}$ in Eq. 21 for $t_{0}^{c}=1 \mathrm{~h}$, i.e. the scaling behaviour for $t_{0}^{c}=0.1 \mathrm{~h} ; 0.5 \mathrm{~h} ; 1 \mathrm{~h} ; 10 \mathrm{~h}$ is shown in Fig. 3 .

While initial scaling depends on the initial curing time (see Eq. 12), it is noted that the late asymptotic form is independent of $t_{0}^{c}$ (see Eq. 13). This says that the prefactor in Eq. 21 is independent of the initial curing time, exhibiting only the temperature dependence of the grain boundary diffusivity and width, and the chemical potential difference, determined by gas and alloy compositions. Extracting this prefactor from finite time experiments, however, requires knowledge of initial conditions related to sample preparation for which there is limited control. By employing $t_{0}^{c}$, the initial conditions may be compensated for, allowing the prefactor to be extracted from finite time exposure experiments. Moreover, there are cases where long-term exposures may not even be feasible. One example is the oxide scaling on zirconium alloys by water, for which the barrier oxide becomes reset by repeated breakdowns, empirically found to follow intermittent 'cube-root kinetics' in line with the present superparabolic-cubic model [23].

The ability to analyse scaling by employing the superparabolic-cubic model to mass gain experiments at 1200 and $1250{ }^{\circ} \mathrm{C}$ [17] is convincingly established in Fig. 4. Furthermore, usefulness as analytic tool is emphasized by addressing what has been long known regarding the deviation from parabolic oxide growth in the alumina formers, it not necessarily being a sign of transient oxide formation (compare, for example, [24]), and that a near-cubic time dependence may indeed be understood to represent an ideal scaling behaviour [22]. From that study, we determine the onset of superparabolic-cubic behaviour to less than $10 \mathrm{~h}$ into the exposure of PM2000 at $1200{ }^{\circ} \mathrm{C}$ (see Fig. 5). 


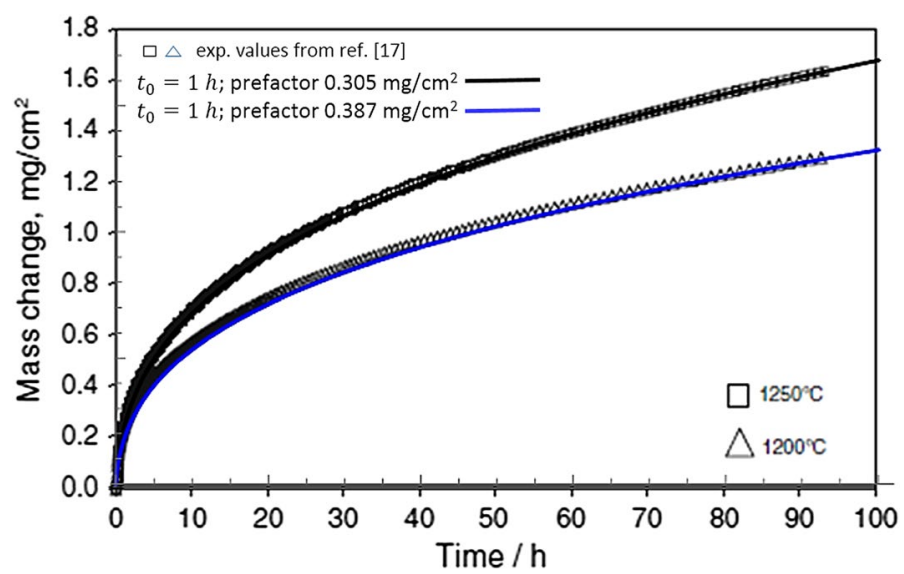

Fig. 4 Mass gain curves from [17] obtained at 1200 and $1250{ }^{\circ} \mathrm{C}$ described by the superparabolic-cubic model for $t_{0}^{c}=1 \mathrm{~h}$ with prefactors $0.305 \mathrm{mg} / \mathrm{cm}^{2}$ and $0.387 \mathrm{mg} / \mathrm{cm}^{2}$, respectively

Moving on, the potential usefulness of the superparabolic-cubic model is further established by revisiting the study on FeCrAlY material [18]. That study considers exposure temperatures at 1200,1250 and $1300{ }^{\circ} \mathrm{C}$, for $20 \% \mathrm{O}_{2}$ in $\mathrm{Ar}$ (oxidizing conditions), as well as $4 \% \mathrm{H}_{2}, 7 \% \mathrm{H}_{2} \mathrm{O}$ in $\mathrm{Ar}$ (reducing conditions). Good agreements with the superparabolic-cubic scaling model are obtained in both cases (see Fig. 6a, b). Employing the equilibrium data of Young et al. [18] concerning oxygen activities at metal-oxide interface and at the oxide-gas interface, slightly modified values of $\delta_{\mathrm{GB}} D_{\mathrm{GB}}$ as compared to that study are obtained for both the oxidizing and the reducing conditions (see Fig. 6c).

Moreover, ability to track differences in alloy composition to their scaling rates is demonstrated by considering the mass gain curves of $\mathrm{FeCrAl}$ and alloy MA 956 at $1100{ }^{\circ} \mathrm{C}$ [1] (see Fig. 7). Agreement with experiment is obtained only for the superparabolic-cubic model. Indeed, while alloy MA 956 quickly enters the cubic asymptote, the $\mathrm{FeCrAl}$ apparently employs a longer initial curing time, thus extending the time spent between initial $X \propto t^{\frac{2}{3}}$ and late $X \propto t^{\frac{1}{3}}$ (compare Eqs. (12), (13) and (19f)).

Care must be taken, however, not to base conclusions regarding scaling processes on agreement with one model alone, and especially so if the two incompatible models appear to describe the experiment well. One such case comprises the recent $\mathrm{CoCrAl}(\mathrm{Y})$ study (air, $900{ }^{\circ} \mathrm{C}$ ) [25]. Here, excellent agreement with experiment is obtained for the superparabolic-cubic model for $t_{0}^{c}=10 \mathrm{~h}$ (see inset in Fig. 8). For reference, $t_{0}^{c}=1 \mathrm{~h} ; 20 \mathrm{~h}$ are also included in Fig. 8a. The large curing time $\left(t_{0}^{c}=10 \mathrm{~h}\right)$ compared to that at $1200-1300{ }^{\circ} \mathrm{C}\left(t_{0}^{c}=1 \mathrm{~h}\right)$ may be owing to the lower temperature. We note that the parabolic-logarithmic model, according to Eq. (22), also provides excellent agreement for the duration of the $250 \mathrm{~h}$ exposure experiment (see Fig. 8e). Arbitrarily, for this model the rate of grains coarsening is taken to be $1.0 \mathrm{~nm} / \mathrm{h}$ throughout the presentation. At long times, however, the logarithmic function detaches from the superparabolic-cubic model as expected (see Fig. 8d). In as 
Fig. 5 Deviation from parabolic model for oxide scaling on PM2000 at $1200{ }^{\circ} \mathrm{C}$ from [22]. Superparabolic-cubic model (red dashed). Deviation between model and experiment emerges at $t<9 \mathrm{~h}$ for $t_{0}^{c}=1 \mathrm{~h}$; prefactor $=0.245 \mathrm{mg} / \mathrm{cm}^{2}$ (Color figure online)
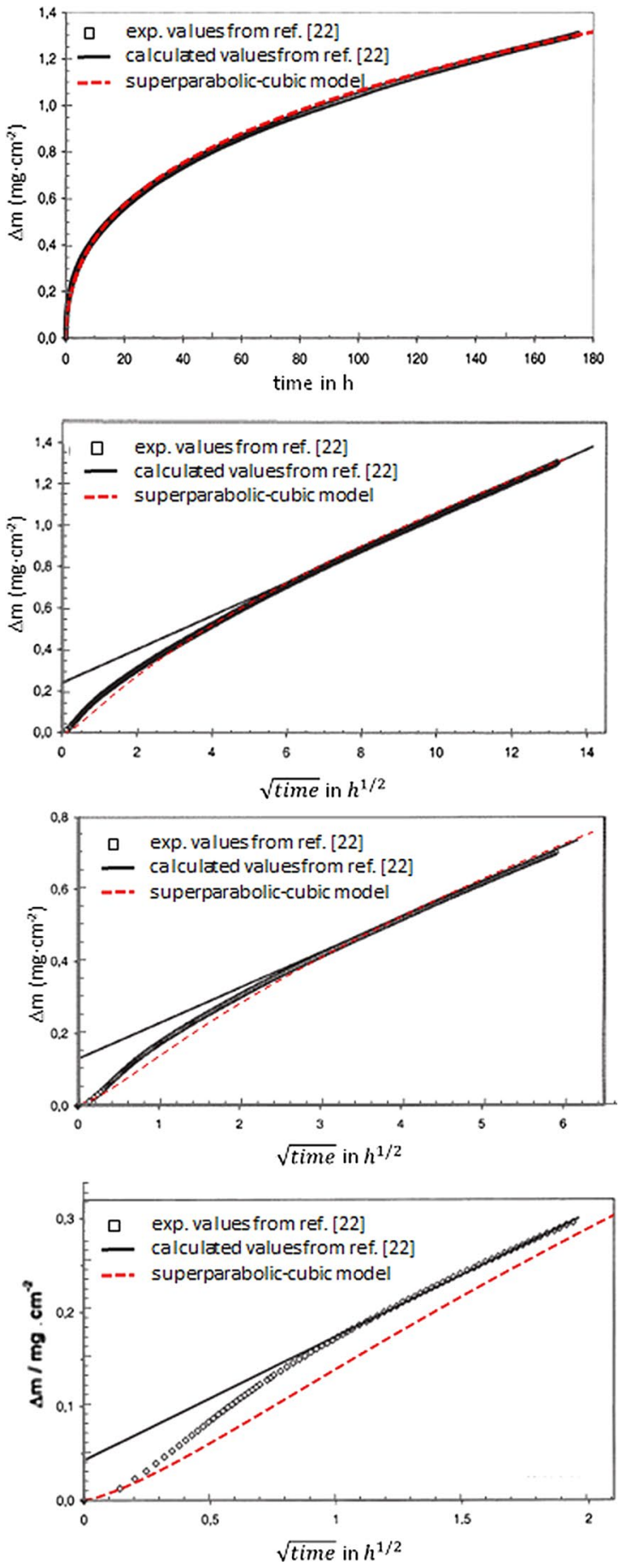
(a)

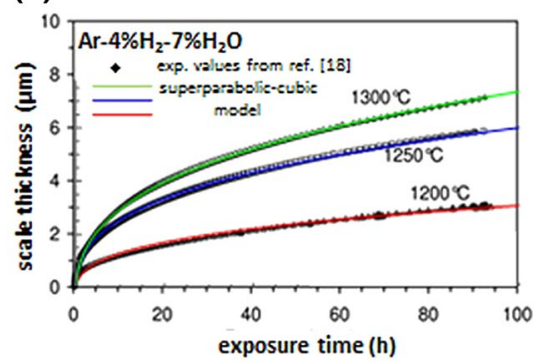

(b)

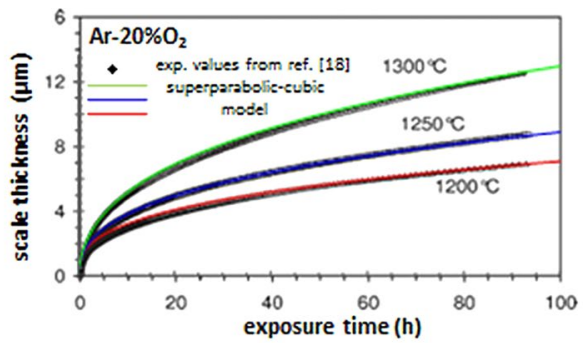

(c)

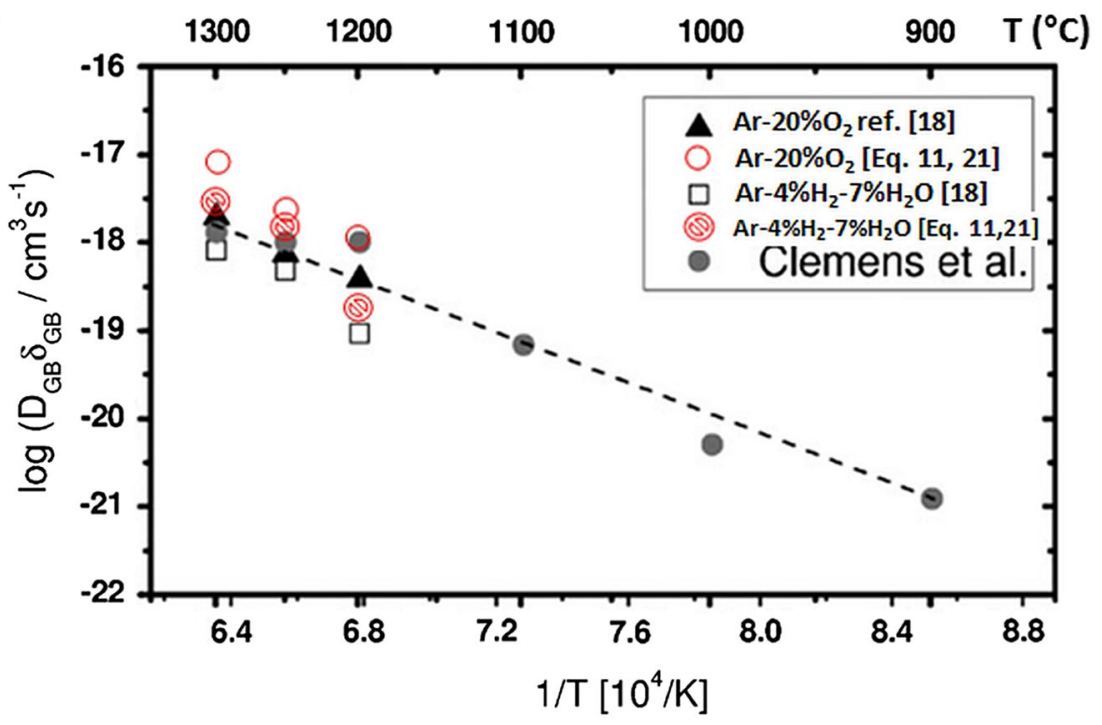

Fig. 6 Exposures in $\mathrm{Ar}-4 \% \mathrm{H}_{2}-7 \% \mathrm{H}_{2} \mathrm{O}$ as well as in $\mathrm{Ar}-20 \% \mathrm{O}_{2}$ at 1200,1250 and $1300{ }^{\circ} \mathrm{C}$ [18] are described, respectively, by the superparabolic-cubic model for prefactors and $t_{0}^{c}$ in parentheses, a $0.7 \mu \mathrm{m}$ $(0.60 \mathrm{~h}) ; 1.35 \mu \mathrm{m}(0.40 \mathrm{~h}) ; 1.7 \mu \mathrm{m}(1.10 \mathrm{~h})$, and b $1.55 \mu \mathrm{m}(0.05 \mathrm{~h} ; 2.00 \mu \mathrm{m}(0.40 \mathrm{~h}) ; 3.03 \mu \mathrm{m}(1.4 \mathrm{~h})$. c $\delta_{\mathrm{GB}} D_{\mathrm{GB}}$ values are obtained from Eq. 11, (red symbols) by employing $\frac{\Delta \mu}{R T}$ data from Ref. 18 (Color figure online)

much as longer exposure times are required in order to discriminate between the two oxide scaling models, here, we envisage that possible transitions between grains coarsening modes may be observed as well, being either coupled to or decoupled from the rate of oxide scale growth. In view of lack of knowledge regarding the true scaling kinetics in this case, the models may be employed to estimate best-case and worst-case mass gain scenarios.

We conclude this section by considering yet another attempt at discriminating between different possible scaling behaviours, applied to MA 956-an $\mathrm{FeCrAl}(\mathrm{Y}, \mathrm{Ti})$ - at $1100{ }^{\circ} \mathrm{C}$ (cf. [1], see Fig. 9). Indeed, here we find the superparabolic-cubic model to agree well with experiment, while the parabolic-logarithmic form appears to decay too fast. These conclusions may be due to oscillations in the 


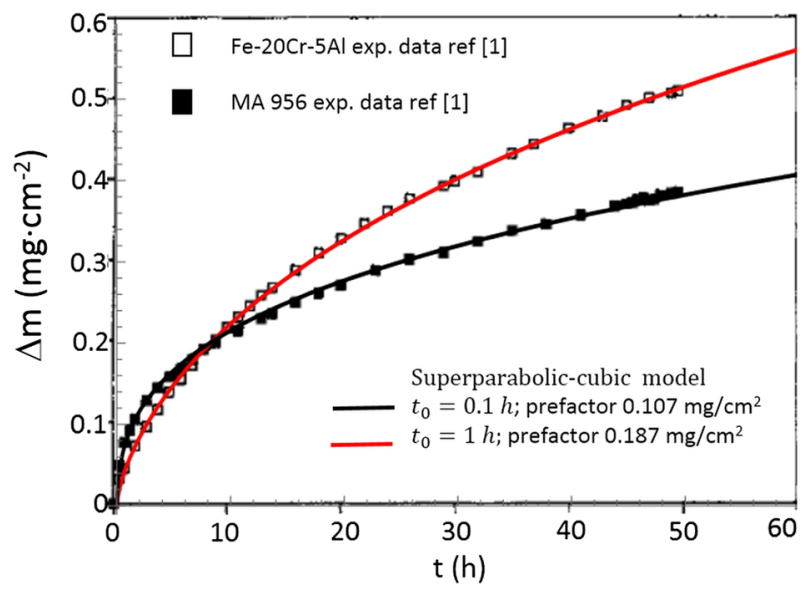

Fig. 7 Comparative mass gain curves for oxidation of MA 956 and an $\mathrm{FeCrAl}$ at $1100{ }^{\circ} \mathrm{C}$, from [1]. Superparabolic-cubic model applied to MA 956: $t_{0}^{c}=0.1 \mathrm{~h}$, prefactor $=0.107 \mathrm{mg} / \mathrm{cm}^{2} ;$ FeCrAl: $t_{0}^{c}=10 \mathrm{~h}$, prefactor $=0.187 \mathrm{mg} / \mathrm{cm}^{2}$
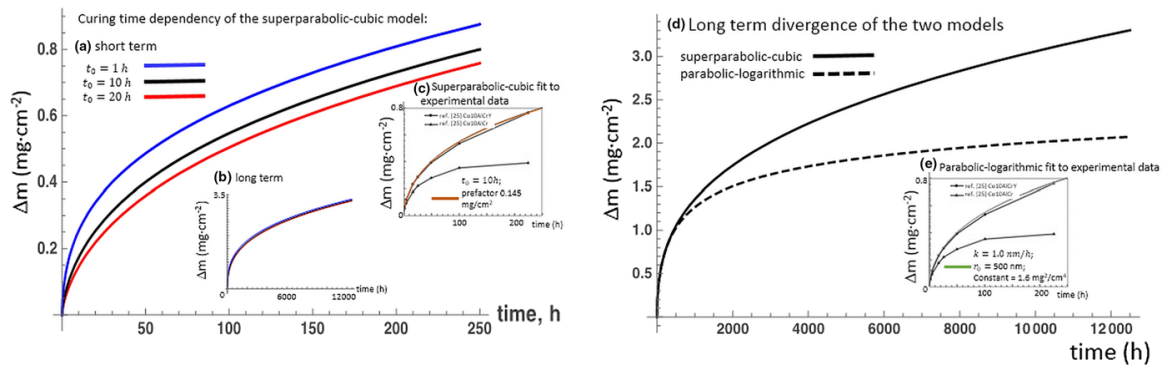

Fig. 8 a Oxide scaling dependent on $1 \mathrm{~h}, 10 \mathrm{~h}$ and $20 \mathrm{~h}$ initial curing times for the superparabolic-cubic model is shown. b Convergence of scale thicknesses irrespective of initial curing times (cf. Equation 12) is demonstrated. c Excellent agreement with experiment at $900{ }^{\circ} \mathrm{C}$ [25] is obtained for $t_{0}^{c}=10 \mathrm{~h}$ and prefactor $0.145 \mathrm{mg} / \mathrm{cm} 2$. d Long-term detachment of superparabolic-cubic model from parabolic-logarithmic model due to different asymptotic forms is demonstrated, upper $X \propto t^{\frac{1}{3}}$ and lower $X \propto \sqrt{\ln (t)}$. Inset: Excellent agreement between parabolic-logarithmic model and experiment is demonstrated, $k=1.0 \mathrm{~nm} / \mathrm{h}, r_{0}=500 \mathrm{~nm}$, constant $=1.60 \mathrm{mg}^{2} / \mathrm{cm}^{4}$

mass gain curve, however. Indeed, oscillations due to fluctuations in the oxidation process between the two modes, i.e. Eqs. 21 and 22, cannot be ruled out. However, the purely parabolic model was refuted already in said study.

\section{Application of Parabolic-Logarithmic Model}

The potential usefulness of the parabolic-logarithmic model, besides being complementary to the superparabolic-cubic model, is explored by addressing three cases. Indeed, its general properties provide a rigorous connection between intuitively related effects of oxide scaling, i.e. those between stress and grains coarsening. 


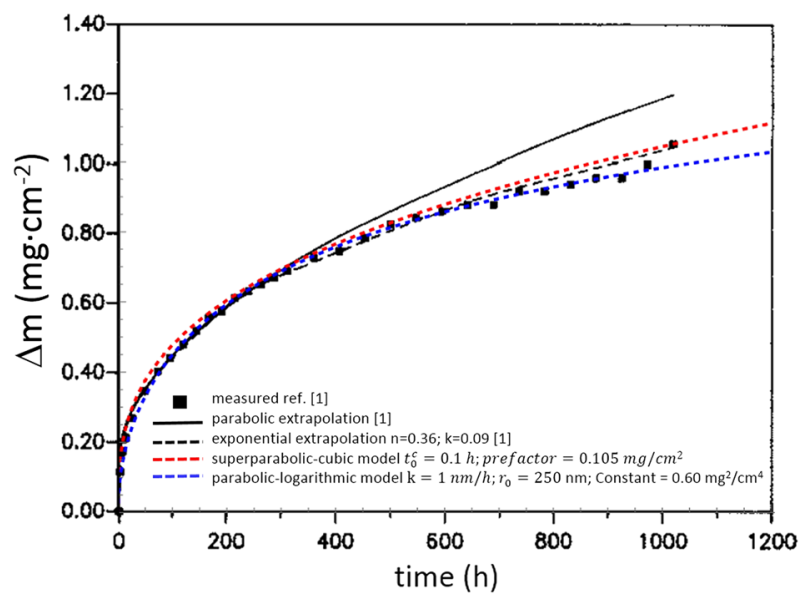

Fig. 9 Comparative analysis of mass gain during oxide growth on MA 956 at $1100{ }^{\circ} \mathrm{C}$. Top: parabolic fit [1]. Red dotted: superparabolic-cubic model, $\left(t_{0}^{c}=0.1 \mathrm{~h}\right.$, prefactor $\left.=0.105 \mathrm{mg} / \mathrm{cm}^{2}\right)$. Blue dotted: parabolic-logarithmic model for $k=1 \mathrm{~nm} / \mathrm{h}, r_{0}=250 \mathrm{~nm}$, and constant: $0.60 \mathrm{mg}^{2} / \mathrm{cm}^{4}$ (Color figure online)

Firstly, we consider the recent $\mathrm{CoCrAl}(\mathrm{Y})$ study [15] mentioned above, which also provides a mass gain curve for the undoped CoCrAl alloy. In this case, excellent agreement is found for the parabolic-logarithmic model, while the superparabolic-cubic model fails. The $t_{0}^{c}$ for the failing superparabolic-cubic model is $0.001 \mathrm{~h}$. And in spite of early access to its cubic asymptote, it is insufficient in describing the

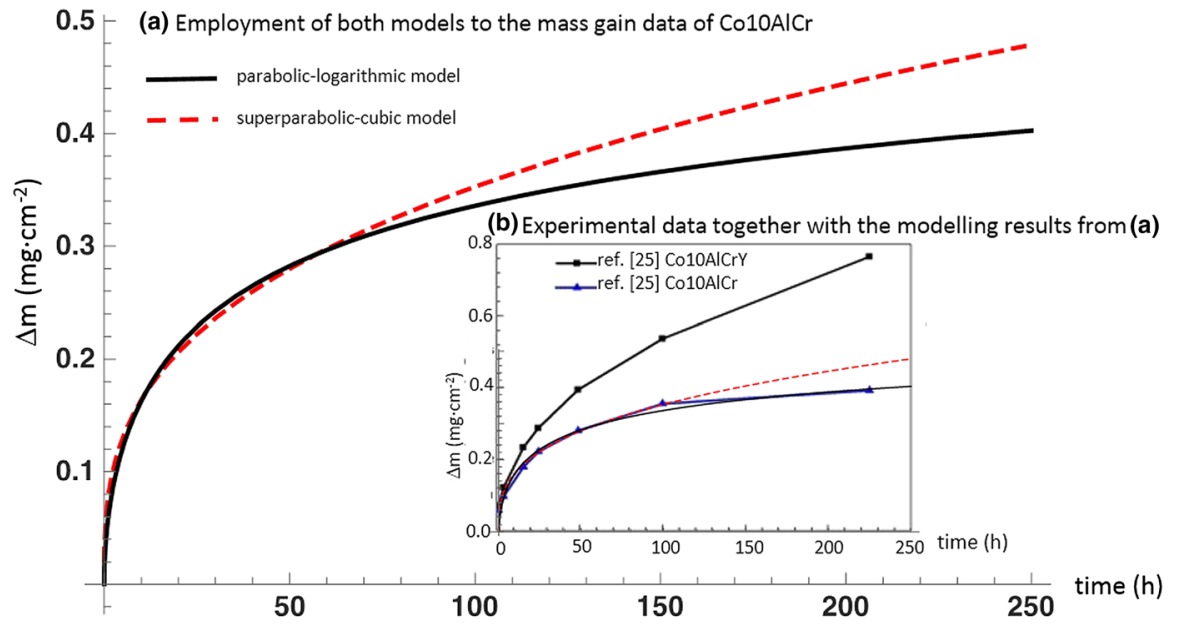

Fig. 10 Comparison of mass gain curves according to parabolic-logarithmic (black line) and superparabolic-cubic (red dashed line) models. Inset: excellent agreement between parabolic-logarithmic model (black line) and experimental observation for $\mathrm{CoCrAl}$ at $900{ }^{\circ} \mathrm{C}$ [25] $k=1 \mathrm{~nm} / \mathrm{h}, r_{0}=17 \mathrm{~nm}$ constant $=0.060 \mathrm{mg}^{2} / \mathrm{cm}^{4}$, while the superparabolic-cubic model is shown to fail (dashed red line) (Color figure online) 
rapidly flattening out of the experimental curve (see Fig. 10). Clearly, this behaviour is easily reproduced by the parabolic-logarithmic model.

A second, possibly related issue is the observed specimen-dependent scaling in 1-mm- and 0.3-mm-thick $\mathrm{FeCrAl}(\mathrm{Y}, \mathrm{Zr})$ alloy samples at $1300{ }^{\circ} \mathrm{C}$ [26]. While the superparabolic-cubic model struggles in describing the $0.3-\mathrm{mm}$ sample, perfect agreements are found for the parabolic-logarithmic model (see Fig. 11a). Notably, the agreements are obtained for the same initial curing times and initial grain widths for the two cases. In particular, the same initial mass gain derivatives tell of the same initial grain boundary density, as captured by the model (see Fig. 11a again). Given that the samples were subjected to the same exposures, these displaying the same experimental grain boundary diffusivities and widths are consistent with the notion that the difference between the two specimens should be found in a specimen-dependent oxygen chemical potential at the alloy-oxide interface, possibly related to $\mathrm{Zr}$ in the alloy. This was proposed to explain the greater mass gain in the 1-mm-thick specimen [26, 27]. A second possibility was that the mismatch in velocities of oxide grain coarsening and oxide thickness growth, assumed by the parabolic-logarithmic model, which would render the two systems different abilities to alleviate scaling-induced stresses, was ruled out it this case [27]. On a similar note, thirdly, oxide scaling on coarse-grained and fine-grained PM2000 alloys was compared at $900{ }^{\circ} \mathrm{C}$ and $1200{ }^{\circ} \mathrm{C}$ [28]. The models developed here are not suitable to describe the observed scaling at $900{ }^{\circ} \mathrm{C}$ owing to prolonged irregular initial oxidation. However, at $1200{ }^{\circ} \mathrm{C}$, while the superparabolic-cubic model describes the fine-grained sample well, and it does less so for the coarse-grained alloy sample. In contrast, excellent agreements with the parabolic-logarithmic model are obtained in both cases (Fig. 11b).

We observe that the initial oxide grain boundary densities of the two specimens come out different, i.e. smaller initial grain width for the coarse-grained alloy

(a)

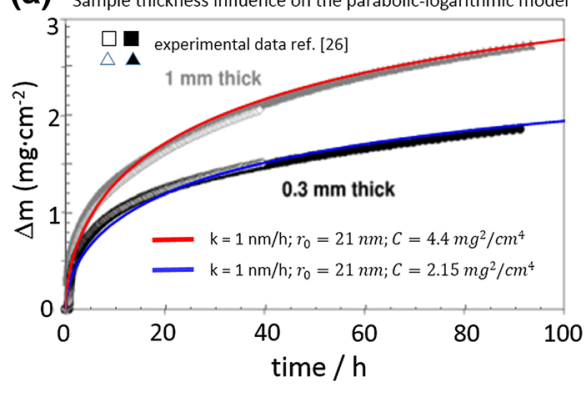

(b) Grain size effect on the models (alloy PM2000)

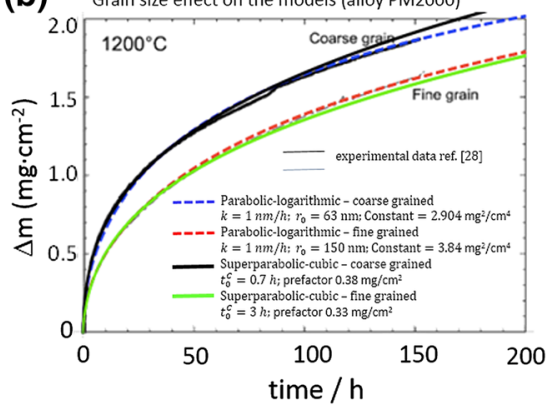

Fig. 11 a Experimental [26] comparison regarding mass gains, for 1-mm and 0.3-mm-thick specimens for one and the same alloy, $\mathrm{FeCrAl}(\mathrm{Y}, \mathrm{Zr})$ at $1300{ }^{\circ} \mathrm{C}$, modelled by the parabolic-logarithmic model for $k=1 \mathrm{~nm} / \mathrm{h}, r_{0}=21 \mathrm{~nm}$ and constants: $4.4 \mathrm{mg}^{2} / \mathrm{cm}^{4}(1 \mathrm{~mm}) ; 2.15 \mathrm{mg}^{2} / \mathrm{cm}^{4}(0.3 \mathrm{~mm})$. b Comparative experimental investigation [28] of coarse-grained and fine-grained PM2000 alloys at $1200{ }^{\circ} \mathrm{C}$ described by the parabolic-logarithmic model: $\left\{\right.$ coarse: $k=1 \mathrm{~nm} / \mathrm{h}, r_{0}=63 \mathrm{~nm}, 2.904 \mathrm{mg}^{2} / \mathrm{cm}^{4}$ (blue dashed) ; \{fine: $k=1 \mathrm{~nm} / \mathrm{h}, r_{0}=150 \mathrm{~nm}, 3.84 \mathrm{mg}^{2} / \mathrm{cm}^{4}$ (red dashed)\}; described by the superparabolic-cubic model: $\left\{t_{0}^{c}=0.7 \mathrm{~h}\right.$; prefactor $=0.38 \mathrm{mg} / \mathrm{cm}^{2}$ (coarse: black full): $\left\{t_{0}^{c}=3 \mathrm{~h}\right.$; prefactor $=0.33 \mathrm{mg} / \mathrm{cm}^{2}($ fine: green full) $\}$ (Color figure online) 
$r_{0}=63 \mathrm{~nm}$ as compared to the $r_{0}=150 \mathrm{~nm}$ for the fine-grained alloy. This is in turn reflected in higher initial mass gain rate in case of the coarse-grained alloy (see Fig. 11b). It is tempting to suggest preference for the parabolic-logarithmic model, particularly for the coarse-grained PM2000, to reflect the fact that initially, the oxide grains coarsening rate cannot keep up with the rate of scale growth. It is conceivable that the ability to alleviate stress by allowing for formation of voids and pores at the moving alloy-oxide interface (cf., for example, Ref. [25]) is indeed a common feature when parabolic-logarithmic scaling is observed. Such scavenging processes would be symptomatic for the residual (electro-)chemical potential gradients owing to the rapid slowdown of oxide growth as represented by the effective 'spring force' attenuated oxide scaling (cf. Eqs. 3 and 8c).

\section{'Effective' Grain Boundary Density in Oxide Scaling}

The invaluable impact of electron microscopy in present days oxidation studies speaks for itself. Indeed, in the present study, grains coarsening has been employed as a conceptual tool for explaining thermogravimetric curves, while resorting to microscopy studies in the literature to provide complementary evidence for the claim. However, the true power of the thermogravimetric (TG) analysis is in that it actually monitors the oxidation process, and while microscopy tells of what has happened, the relevance of the images for the oxidation process is by necessity only implicit. The comparative study of chromia scaling of $\mathrm{Ni} 25 \mathrm{Cr}$ at $1000{ }^{\circ} \mathrm{C}$ in dry and wet $\mathrm{N}_{2}-1 \% \mathrm{O}_{2}$ [29] is particularly instructive. TG reports dramatic suppression of oxidation rate under humid conditions, while microscopy reports no change in grain size distribution. Fundamentally, transport paths may be sealed either by explicit grains coarsening or by the 'clogging' of grain boundaries. Indeed, hydrogen residing in interstitial sites in the form of hydroxide ions was suggested in [29] to restrict transport in the crystalline grain boundaries. And yet both grains coarsening models developed here are able to describe the early subparabolic signatures of the TG data provided in [29] (see Fig. 12a, compare Fig. 8c-e). We arrive at the understanding that the continuous loss of 'effective' grain boundary density as reflected in the transport properties disagrees with a quasi-static poly-crystalline oxide microstructure. The apparent grain growth, which emerges from the TG, is taken to imply that easy ion diffusion paths continuously become sealed off by the hydrolysis process, e.g. by 'glassy' subnanointerfaces between grains being formed. This straightforward interpretation for wet conditions is contrasted by the dry exposures. Thus, the parabolic-logarithmic model cannot describe the observed 'superparabolic' scaling (see Fig. 12b). For this, only the superparabolic-cubic model is applicable (compare Eq. 12 (superparabolic) and Eq. 9b (parabolic)). Thus, in case of 1-mm specimen, $10 \mathrm{~h}$ curing time is employed (see Fig. 12b). Hence, the 'waist' in the grains coarsening evolution as function of scaling ends up at $12.5 \mathrm{~h}$ (compare Fig. $2 \mathrm{c}$ and Eq. 18b). The impact of this comparatively large curing time in relation to the 25-h duration of the experiment is not insignificant. This is further accentuated in the modelling of dry oxidation of the $0.25-\mathrm{mm}$ specimen where the $t_{0}^{c} \gg t$ limit becomes even more relevant (see Fig. 12b again). Here the 'waist' is at 125 h, i.e. $t=1.25 t_{0}^{c}$ for $t_{0}^{c}=100 \mathrm{~h}$. 

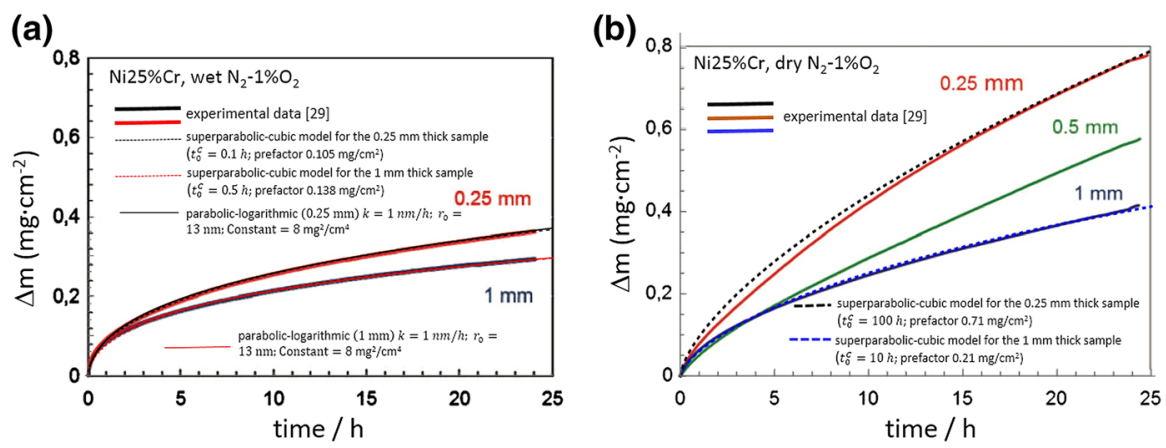

Fig. 12 a Experiment from [29], wet $\mathrm{N}_{2}-1 \% \mathrm{O}_{2}$. Full lines and dotted lines refer to the superparaboliccubic and the parabolic-logarithmic models, respectively. Inverted colours: red for $1 \mathrm{~mm}$ and black for $0.25-\mathrm{mm}$ specimens for contrast. Parabolic-logarithmic model (full lines) for $k=1 \mathrm{~nm} / \mathrm{h}, 0.25 \mathrm{~mm}$ : $r_{0}=13 \mathrm{~nm}$ and constants: $8 \mathrm{mg}^{2} / \mathrm{cm}^{4} ; 1 \mathrm{~mm}: r_{0}=23 \mathrm{~nm}$ and constants: $18 \mathrm{mg}^{2} / \mathrm{cm}^{4}$. Superparaboliccubic model (dotted lines): $0.25 \mathrm{~mm}:\left\{t_{0}^{c}=0.1 \mathrm{~h}\right.$; prefactor $=0.105 \mathrm{mg} / \mathrm{cm}^{2} ; 1 \mathrm{~mm}:\left\{t_{0}^{c}=0.5 \mathrm{~h}\right.$; prefactor $\left.=0.138 \mathrm{mg} / \mathrm{cm}^{2}\right\}$. b Experiment from [23], dry $\mathrm{N}_{2}-1 \% \mathrm{O}_{2}$. Superparabolic-cubic model (black dotted lines): $0.25 \mathrm{~mm}$ : $\left\{t_{0}^{c}=100 \mathrm{~h}\right.$; prefactor $=0.71 \mathrm{mg} / \mathrm{cm}^{2} ; 1 \mathrm{~mm}:\left\{t_{0}^{c}=10 \mathrm{~h}\right.$; prefactor $\left.=0.21 \mathrm{mg} / \mathrm{cm}^{2}\right\}$. Parabolic-logarithmic model not applicable. $0.5 \mathrm{~mm}$ could not be modelled (see text) (Color figure online)

While such scaling phenomenology at early stages is commonly hidden or deemed inaccessible, the superparabolic-cubic model offers a semi-quantitative overall contextual understanding. Failing to describe even qualitatively the scaling process of the $0.5-\mathrm{mm}$ specimen under dry conditions may point at critical phenomena also present at $1000{ }^{\circ} \mathrm{C}$, and not captured by the simple model. Having said this, it is gratifying to note how well the phenomenological second-order kinetics approach of Fehlner and Mott [16] as realized in the superparabolic-cubic model is able to capture essential aspects of the underlying complexities of the chromia formers, mainly under wet conditions but to some extent also in dry.

\section{Concluding Remarks}

A simple, yet comprehensive approach to oxide scaling was described with emphasis on the impact of oxide grains coarsening on the oxidation of high-temperature alloys. It is based on two fundamental assumptions: Firstly, the scaling process is governed by grain boundary diffusion alone, and secondly, grains coarsening occurs exclusively at the moving oxide front. First- and second-order kinetics in loss of grains boundary density were considered, but in the light of said assumptions, only the latter was deemed physical. Expressions for oxide scaling were formulated, and essential aspects emanating from the lateral grains coarsening rate being connected to- - or disconnected from-the rate of scale growth were articulated. Asymptotic forms of the two mutually excluding models were deduced and employed to discuss experimental scaling studies in the literature. The potential usefulness was illustrated by providing new estimated values of $\delta_{\mathrm{GB}} D_{\mathrm{GB}}$ from experimental scaling experiments. The term 'effective' grains coarsening was introduced for chromia scaling in 
wet and dry $\mathrm{N}_{2}-1 \% \mathrm{O}_{2}$ in order to explain subparabolic suppression of oxide growth in case of the former in spite of little difference in grain size distribution between the two. The phenomenology inherent in the superparabolic-cubic model was employed to articulate early scaling of chromia in dry $\mathrm{N}_{2}-1 \% \mathrm{O}_{2}$.

Arguably, the degree of synchronization between rates of scale growth and oxide grains coarsening may change during scaling, early between superparabolic $X \propto t^{\frac{2}{3}}$ or parabolic $X \propto t^{\frac{1}{2}}$, and late between cubic $X \propto t^{\frac{1}{3}}$ or logarithmic $X \propto \sqrt{\ln (t)}$. Yet, it should be born in mind that, inasmuch as both grains coarsening models display late subparabolic scale growth, in the absence of scale breakdown the parabolic lattice diffusion channel is expected to eventually prevail. Having said this, scavenging processes driven by residual (electro-)chemical potential gradients might disallow this limit to be reached (compare, for example, the cyclic barrier oxide breakdown prevalent for zirconia formers [30, 31]).

In summary, a conceptually straightforward and practical modelling approach is provided that allows discrimination between different oxidation mechanisms and the extraction of scaling kinetics information from mass gain experiments, benefiting future materials development, not least by identifying deviations from the ideal. Moreover, the ability to connect oxide scaling kinetics with activation energies for diffusion offers a bridge between experiment and first-principles atomistic model studies, to allow the pinpointing of the decisive transport channels for future tailored control.

Acknowledgements This work was carried out within the Swedish High Temperature Corrosion Center (HTC).

Open Access This article is distributed under the terms of the Creative Commons Attribution 4.0 International License (http://creativecommons.org/licenses/by/4.0/), which permits unrestricted use, distribution, and reproduction in any medium, provided you give appropriate credit to the original author(s) and the source, provide a link to the Creative Commons license, and indicate if changes were made.

\section{References}

1. W. J. Quadakkers, Materials and Corrosion 41, 1990 (659).

2. J. S. Benjamin, Metallurgical Transactions 1, 1970 (2943).

3. J. A. Nesbitt, Journal of The Electrochemical Society 136, 1989 (1511).

4. J. Young, in Corrosion Series, ed. Y. D. John (Elsevier Science, New York, 2008), p. 315.

5. T. Liu, C. Wang, H. Shen, W. Chou, N. Y. Iwata and A. Kimura, Corrosion Science 76, 2013 (310).

6. F. H. Stott, G. C. Wood and J. Stringer, Oxidation of Metals 44, 1995 (113).

7. D. Naumenko, B. A. Pint and W. J. Quadakkers, Oxidation of Metals 86, 2016 (1).

8. S. Chevalier, Developments in High Temperature Corrosion and Protection of Materials, (Woodhead Publishing, New York, 2008), p. 290.

9. A. H. Heuer, T. Nakagawa, M. Z. Azar, D. B. Hovis, J. L. Smialek, B. Gleeson, N. D. M. Hine, H. Guhl, H. S. Lee, P. Tangney, W. M. C. Foulkes and M. W. Finnis, Acta Materialia 61, 2013 (6670).

10. J. L. Smialek, Metallurgical Transactions A 22, 1991 (739).

11. N. Mortazavi, C. Geers, M. Esmaily, V. Babic, M. Sattari, K. Lindgren, P. Malmberg, B. Jönsson, M. Halvarsson, J. E. Svensson, I. Panas and L. G. Johansson, Nature Materials 17, 2018 (610).

12. G. Tammann and W. Köster, Zeitschrift für Anorganische und Allgemeine Chemie 123, 1922 (196).

13. C. Wagner, Journal of The Electrochemical Society 103, 1956 (571). 
14. E. W. Hart, Acta Metallurgica 5, (10), 1957 (597).

15. W. W. Smeltzer, R. R. Haering and J. S. Kirkaldy, Acta Metallurgica 9, 1961 (880).

16. F. P. Fehlner and N. F. Mott, Oxidation of Metals 2, 1970 (59).

17. D. Naumenko, B. Gleeson, E. Wessel, L. Singheiser and W. J. Quadakkers, Metallurgical and Materials Transactions A 38, 2007 (2974).

18. D. J. Young, D. Naumenko, L. Niewolak, E. Wessel, L. Singheiser and W. J. Quadakkers, Materials and Corrosion 61, 2010 (838).

19. D. J. Young, in Corrosion Series, ed. D. J. Young (Elsevier, New York, 2016), p. 367.

20. C. Geers, V. Babic, N. Mortazavi, M. Halvarsson, B. Jönsson, L.-G. Johansson, I. Panas and J.-E. Svensson, Oxidation of Metals 1, 2017 (1).

21. L. P. H. Jeurgens, W. G. Sloof, F. D. Tichelaar and E. J. Mittemeijer, Journal of Applied Physics 92, 2002 (1649).

22. W. J. Quadakkers, D. Naumenko, E. Wessel, V. Kochubey and L. Singheiser, Oxidation of Metals 61, 2004 (17).

23. J. S. Bryner, Journal of Nuclear Materials 82, 1979 (84).

24. D. Naumenko, W. J. Quadakkers, A. Galerie, Y. Wouters and S. Jourdain, Materials at High Temperatures 20, 2003 (287).

25. H. Yu, S. Ukai, S. Hayashi and N. H. Oono, Corrosion Science 127, 2017 (147).

26. V. Kochubey, H. Al-Badairy, G. Tatlock, J. Le-Coze, D. Naumenko and W. J. Quadakkers, Materials and Corrosion 56, 2005 (848).

27. W. J. Quadakkers, P. Huczkowski, D. Naumenko, J. Zurek, G. H. Meier, L. Niewolak and L. Singheiser, Materials Science Forum 595-598, 2008 (1111).

28. P. Pérez, Corrosion Science 44, 2002 (1793).

29. M. Hänsel, E. Turan, V. Shemet, D. Grüner, U. Breuer, D. Simon, B. Gorr, H. J. Christ and W. J. Quadakkers, Materials at High Temperatures 32, 2015 (160).

30. M. Lindgren and I. Panas, Oxidation of Metals 87, 2017 (355).

31. M. Lindgren, C. Geers and I. Panas, Journal of Nuclear Materials 492, 2017 (22). 